\title{
Wavelet-based multispectral face recognition
}

\author{
LIU Dian-ting ${ }^{1 *}$, ZHOU Xiao-dan ${ }^{2}$, and WANG Cheng-wen ${ }^{3}$ \\ 1. CAD, CG \& Network Laboratory, School of Mechanical Engineering, Dalian University of Technology, Dalian 116024, \\ China; \\ 2. School of Electronic and Information Engineering, Dalian University of Technology, Dalian 116024, China; \\ 3. Department of Labor Security, Xiangying Town Government, Dalian 116111,China.
}

(Received 24 May 2008)

\begin{abstract}
This paper proposes a novel wavelet-based face recognition method using thermal infrared (IR) and visible-light face images. The method applies the combination of Gabor and the Fisherfaces method to the reconstructed IR and visible images derived from wavelet frequency subbands. Our objective is to search for the subbands that are insensitive to the variation in expression and in illumination. The classification performance is improved by combining the multispectal information coming from the subbands that attain individually low equal error rate. Experimental results on Notre Dame face database show that the proposed wavelet-based algorithm outperforms previous multispectral images fusion method as well as monospectral method.
\end{abstract}

CLC numbers: TP391.4 Document code: A Article ID: 1673-1905(2008)05-0384-3

DOI 10.1007/s11801-008-8049-8

As a hot research area of biometric technologies during the past several years, face recognition has received considerable progress in controlled environments, where the lighting, pose, background, and the quality of images are well controlled ${ }^{[1]}$. But in uncontrolled environments, random lighting sources from different directions significantly change visual appearances and influence the representation of the visible spectrum images. To cope with the challenges posed by visible spectral images, thermal infrared images have been used as complements of visible light images for face recognition. Long-wave IR or thermal images, captured in the range of $8-12 \mu \mathrm{m}$, represent the heat pattern of the object and are invariant to illumination and expression ${ }^{[2]}$. Compared with other spectral bands below the visible spectra such as $\mathrm{X}$-rays and ultraviolet radiation, thermal IR spectrum has no harm to the human body, and therefor it can be employed in face recognition applications ${ }^{[3]}$.

Recently, wavelet transform has been used in many studies on face recognition ${ }^{[4-6]}$. In Ref.[4], several wavelet families are used in order to extract multiple subband images and the application of Laplacian Eigen-Maps (LEM) on the best subbands chosen by running sequential forward floating selection (SFFS) obtains a low equal error rate. Besides, in Ref.[5] the low-frequency subband of the wavelet transform is retained and then this subband is reduced by using a linear projection to improve the performance. The method of com-

\footnotetext{
* E-mail: diantingliu@yahoo.com.cn
}

bining eigenface with different wavelet subbands is proposed in Ref.[6]. In this study, different approximation and details components resulting from 4-level wavelet decomposition are used as the input of principal component analysis (PCA). In spite of these previous studies, the applications of wavelet transform for face recognition do not end here.

Beginning with the idea that different modal face images bring different information, we study the effects of the combination among different subbands extracted from IR and visible face images. In the paper, we first evaluate the individual performance of different subbands extracted from IR and visible images respectively and then choose the best performing subband of IR and visible face images for further feature representation and discriminant analysis. As for the performance evaluation we adopt the equal error rate (EER) ${ }^{[10]}$. The EER represents the intrinsic error of the system, and it is usually considered as a good indicator of the performance of an identification system. The flowchart of the proposed method is shown in Fig.1.

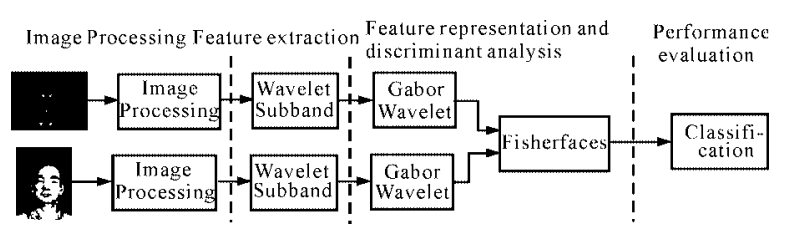

Fig.1 Steps in the proposed wavelet-based multispectral algorithm

Wavelet transform provides powerful signal analysis tools, 
which are widely used in feature extraction, image compression and denoising applications. Since, continuous wavelet transform (CWT) provides redundant information and requires lots of computation, discrete wavelet transform (DWT) is generally preferred. The two-dimensional wavelet transform is performed by consecutively applying one-dimensional wavelet transform to the rows and columns of the two-dimensional data. This paper applies visible and IR images in the discrete wavelet transform domain. Assuming the multispectral images are of the same sizes, IR and visible images are transformed into a set of approximation and detail components at a predefined analysis level using the DWT. In the first level, the original image is decomposed into four subbands: the scaling component containing global low-pass information (A1) and three wavelet components corresponding, respectively, to the horizontal details (H1), vertical details (V1) and diagonal details (D1). Consequently A1 is proceeded to decompose, yielding four subband images-A2, H2, V2 and D2. Fig. 2 shows an example (visible image) of 2-level DWT decompositions using the discrete Meyer wavelet.

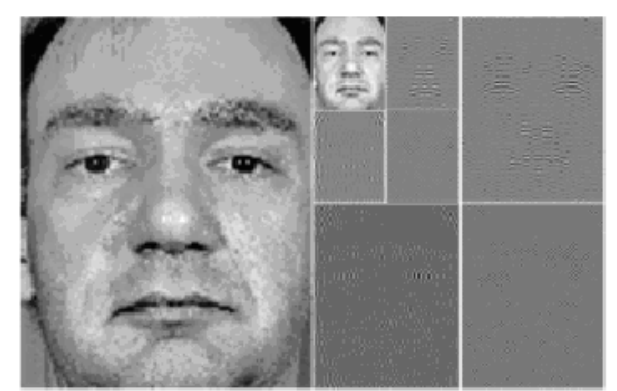

Fig.2 2D 2-level discrete Meyer wavelet decomposition of a given visible image

The characteristics of the Gabor wavelets, especially for frequency and orientation representations, are similar to those of the human visual system, and they have been found to be appropriate for texture representation and discrimination.

A family of Gabor filters is defined as follows:

$$
\begin{aligned}
& \boldsymbol{\psi}_{\mathbf{u}, \mathbf{v}}(x)=\left(\left\|\boldsymbol{k}_{\mathbf{u}, \mathbf{v}}\right\|^{2} / \sigma^{2}\right) \exp \left(-\left\|\boldsymbol{k}_{\mathbf{u}, \mathbf{v}}\right\|^{2}\|x\| / 2 \sigma^{2}\right) \times \\
& \left(\exp \left(i \boldsymbol{k}_{\mathbf{u}, \mathbf{v}} \cdot x\right)-\exp \left(-\sigma^{2} / 2\right)\right),
\end{aligned}
$$

where $u$ and $v$ define the orientation and scale of the Gabor filters, respectively and the wave vector $\boldsymbol{k}_{u, v}$ is defined by $\boldsymbol{k}_{u, v}=\boldsymbol{k}_{v} \exp$ $\left(i \varphi_{u}\right)$, where $\left|k_{v}\right|=k_{\max } / f^{v}$, and $\varphi_{u}=(u \pi / 8) \times k_{\max }$ is the maximum frequency, $f$ is the spacing factor between kernels in the frequency domain. In most face recognition application, we use the Gabor filters with the following parameters ${ }^{[8]}$ : five scales $v \in\{0, \cdots, 4\}$; eight orientations $u \in\{0, \cdots, 7\}$; $k_{\text {max }}=\pi / 2 ; f=\sqrt{2} ; \sigma=2 \pi$.

The Gabor wavelet representation of a face image is the convolution of the image with the family of Gabor filters as defined by eg.(1). The convolution of image $I(x)$ and a Gabor filter $\Psi_{u, v}(x)$ can be defined as follows:

$$
G_{\mathbf{u}, \mathbf{v}}(x)=I(x) * \psi_{\mathbf{u}, \mathbf{v}}(x)
$$

where $G_{u, v}(x)$ denotes the convolution result corresponding to the Gabor filter at orientation $u$ and scale $v$. As a result, the image $I(x)$ can be represented by a set of Gabor wavelet coefficients $\left\{G_{u, v}(x), v=0, \cdots, 4 ; u=0, \cdots, 7\right\}$.

The Fisherfaces method ${ }^{[7]}$ is commonly used for dimensionality reduction in computer vision, face recognition, etc. The algorithm first projects the training images via PCA to a $n-c$ dimensional space with respect to data compression. Then taking into account the recognition (discrimination) aspect, it performs Fisher's linear discriminant to obtain $c$ - 1 dimensional feature vectors, where $n$ is the number of training images, and $c$ is the number of classes.

We present and assess the advantage of the proposed wavelet-based multispectral face recognition scheme, using a data set from Notre Dame face database ${ }^{[9]}$. Specially we use 1968 pairs of IR and visible face images corresponding to $63 \mathrm{sub}-$ jects with variations in expression, lighting, and time lapse. First, the centers of the eyes of an image are manually detected. Then rotation and scaling transformations align the centers of the eyes to predefined locations. Finally, the face image is cropped to the size of $128 \times 100$ to extract the facial region, which is further normalized to zero mean and unit variance. Fig. 3 shows some example images used in our experiments that are already cropped to the size of $128 \times 100$.

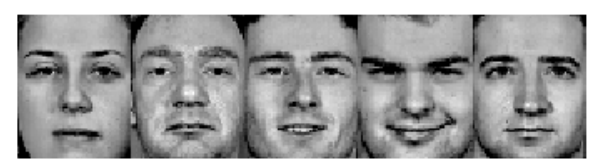

(a)

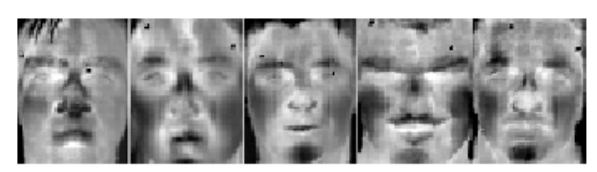

(b)

Fig.3 The Notre Dame images used in our experiments that are already cropped to the size of $128 \times 100$

The discrete Meyer wavelet is used in the study, which is proven to have better verification performance among several wavelet families in a couple of public face database ${ }^{[4]}$. 
In the following experiments, we adopt $k$-fold crossvalidation. First, we averagely divide the dataset into $k$ folds (we let $k=8$ in the experiments). Then for each of $k$ experiments, we use $k-1$ folds for training and the remaining one for testing. The true error is estimated as the average error rate.

For the comparison purpose, we first have tested the individual performance of different subbands with three level of decomposition using discrete Meyer wavelet on IR and visible image respectively. The face recognition performance of these subbands is listed in Tab.1, which shows that the best performance is achieved using the low-pass information at the first level of decomposition.

From the analysis of Tab.1, we observe that the low-pass information on the wavelet transform is the most useful feature set for our verification problem. It is interesting to note that the low-pass information at the first level of decomposition even performs slightly better than the original image, probably because low frequency subband is the smoothed version of the original image and it helps to reduce the influence of noise.

Tab.1 Average EER (in percentage) obtained in different wavelet subbands ( $A$ : low-pass information; $H$ : horizontal details; $V$ : vertical details; the following number is the level of decomposition)

\begin{tabular}{ccc}
\hline & IR & Visible \\
\hline Original & 21.73 & 4.68 \\
A1 & 20.95 & 4.34 \\
A2 & 23.22 & 6.16 \\
A3 & 29.04 & 0.99 \\
H2 & 45.53 & 8.64 \\
H3 & 35.09 & 9.41 \\
V2 & 44.76 & 15.13 \\
V3 & 35.86 & 20.33 \\
\hline
\end{tabular}

Our next series of experiments, performed using the best performing subbands (the low-pass information at the first level) obtained in the above experiments, show that the combination of IR and visible features may enhance the discrimination power of face images.

Fig. 4 shows the comparative face recognition performance of the original visible images, image fusion in the wavelet domain ${ }^{[3]}$ and the proposed wavelet-based fusion method. The proposed method performs better than the other two methods. In particular, when a fixed false match rate (FMR) is given, usually lower than EER (e.g. $F M R=1 \%$ ), the new method achieves a lower false non-match rate (FNMR) than the other two methods.

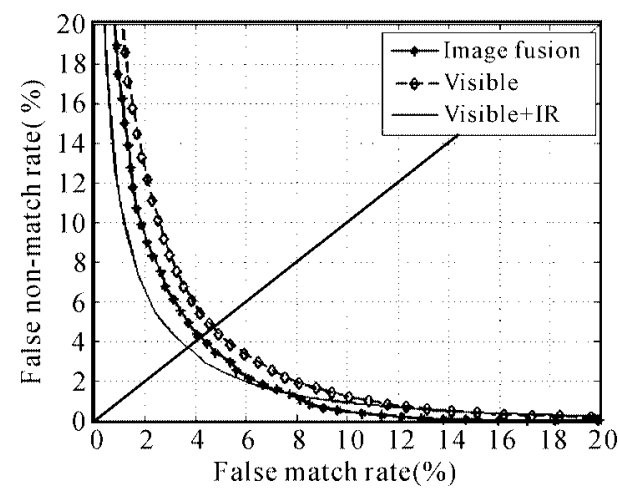

Fig.4 DET-curve on the Notre Dame face database

In conclusion, we have investigated different frequency subbands obtained by wavelet transform with different levels of decomposition. Briefly, it has been observed that the frequency subband containing approximation component is successful in both IR and visible face images. To enforce diversity we have combined IR and visible Gabor features. The experimental have results obtained on Notre Dame face database are encouraging: we show that the EER gained by the combination of the best performing multispectral subbands is lower than that obtained by image fusion in the wavelet domain and the original visible image on the gray values. As future work we want to use a feature selection approach to search few features from IR and visible images for the combination.

\section{References}

[1] W. Zhao, R. Chellappa, P. Phillips, and A. Rosenfeld, ACM Computing Surveys, 35 (2003), 399.

[2] R. Singh, M. Vatsa, and A. Noore, Pattern Recognition, 41 (2008), 880.

[3] S.G. Kong, J. Heo, F. Boughorbel, Y. Zheng, B.R. Abidi,. A. Koschan, M. Yi, and M.A. Abidi, International Journal of Computer Vision, 71 (2007), 215.

[4] L. Nanni and A. Lumini, Pattern Recognition Letters, 29 (2008), 343.

[5] H.K. Ekenel and B. Sankur, Image and Vision Computing, 23 (2005), 469.

[6] Y. Ma and S. Li, Optoelectronics Letters, 2 (2006), 383.

[7] P.N. Belhumeur, J.P. Hespanha, and D.J. Kriegman, IEEE Trans. Pattern Anal. Machine Intell., 19 (2007), 711.

[8] C. Liu and H. Wechsler, IEEE Trans. Image Processing, 11 (2002), 467.

[9] X. Chen, P.J. Flynn, and K.W. Bowyer, Proc. of ACM Workshop on Multimodal User Authentication, 2003, 48.

[10] D. Maio, D. Maltoni, A.K. Jain, and S. Prabhakar, Handbook of Fingerprint Recognition. Springer, New York, 2003, 13. 